\title{
Pharmacological and clinical aspects of immediate release fentanyl preparations: criteria for selection
}

\author{
E J M Kuip, ${ }^{1}$ M L Zandvliet, ${ }^{2}$ R H J Mathijssen, ${ }^{1}$ C C D Van der Rijt ${ }^{1}$
}

In palliative care, pain management is often hampered by episodes of breakthrough pain, characterised by a rapid onset and, on average, duration less than $1 \mathrm{~h}$. Until recently, only immediate release morphine and oxycodon preparations were available for the treatment of these episodes but time until effect is too long for both of these drugs. Recently, immediate release fentanyl products have become available for the treatment of breakthrough pain. These products can be classified as oromucosal and nasal products. Both are absorbed rapidly by the mucosa, although the oromucosally delivered products are partly absorbed by the gastrointestinal tract and therefore reach maximum plasma levels somewhat slower (after 30-90 min) than the nasally delivered products (after $\sim 15 \mathrm{~min}$ ). In clinical placebo controlled studies, all new immediate release fentanyl products were proven to be effective from 15 min after administration in the treatment of breakthrough pain. The first studies comparing immediate release fentanyl with immediate release morphine or oxycodon also found superiority for the new fentanyl products.

\section{Introduction}

In patients with chronic and severe cancer pain, opioids can be used to control the baseline levels of pain by stable systemic exposure via different administration routes. Besides chronic pain, many patients with malignant disease experience breakthrough pain. Breakthrough pain is defined as a transitory exacerbation of pain experienced by the patient who has relatively stable and adequately controlled baseline pain. ${ }^{12}$ It is typically characterised by a rapid onset, mostly within several minutes. Its duration varies widely, but for most patients exacerbation of pain diminishes within $1 \mathrm{~h}^{2}$ For the treatment of breakthrough pain, immediate release morphine and oxycodon

'Department of Medical Oncology, Erasmus University Medical Center, Rotterdam, The Netherlands 2Department of Hospital Pharmacy, Erasmus University Medical Center, Daniel den Hoed Cancer Center, Rotterdam, The Netherlands

Correspondence to DrCCDVan der Rijt, Department of Medical Oncology, Erasmus University Medical Center, Groenehilledijk 301, Rotterdam 3075 EA, The Netherlands; c.vanderrijt@erasmusmc.nl

\section{UNLOCKED}

This paper is freely available online under the BMJ Journals unlocked scheme, see http:// ejhp.bmj.com/info/unlocked.dtl preparations have been used. Until recently, there were no other possibilities for treating breakthrough pain. Both products are effective, but time until the effect is too long. Nowadays, immediate release fentanyl products are available.

Fentanyl is currently a widely used opioid in cancer pain. It is more potent than morphine, highly lipophilic and binds strongly to plasma proteins. ${ }^{3}$ Fentanyl patch for the treatment of continuous (cancer) pain is most often used. For immediate release, there are oromucosal products for transmucosal, buccal and sublingual administration routes. Apart from the oromucosal products, two nasal formulations of fentanyl have been developed. One is formulated with pectin (a polysaccharide obtained from plants) to improve mucosal adhesion while the other does not contain pectin.

In this brief overview, we describe the main pharmacokinetics and clinical aspects of these four administration routes for immediate release fentanyl preparations. These aspects may support selection of the optimal product for individual cancer patients.

\section{Administration routes and pharmacokinetic characteristics As mentioned above, there are various oromucosal products available. One}

is a fentanyl solution on a stick (oral transmucosal fentanyl citrate, Actiq), like a lollipop. By rubbing it over the mucosa for $15 \mathrm{~min}$, fentanyl is totally absorbed. The buccal tablet variants (Breakyl and Effentora) have to be placed between the upper gum and cheek above a molar tooth. Within 30 min it will usually be absorbed by the mucosa. The sublingual fentanyl tablet (Abstral) is a rapidly disintegrating tablet that allows systemic delivery by the oral mucosa.

Fentanyl is also available in nasal sprays (PecFent and Instanyl), where the uptake of fentanyl takes place directly through the nasal mucosa. The spray uses the potential for rapid absorption resulting from the high vascularity and permeability of nasal tissue.

The bioavailability for the oromucosal products varies between $50 \%$ and 70\% (table 1). These relatively low bioavailabilities are explained by partial loss of fentanyl via first pass metabolism following intestinal absorption instead of direct uptake via the oral mucosa. ${ }^{3}$ The bioavailability of nasally delivered fentanyl is much higher ( 90\%). ${ }^{4}$ The small volume of the spray is directly absorbed by the nasal tissue and there is hardly any loss in the gastrointestinal tract by swallowing of fentanyl.

Pharmacokinetic studies in humans have been performed using immediate release fentanyl products. For the oromucosal 


\begin{tabular}{|c|c|c|c|c|c|c|c|c|c|}
\hline \multicolumn{2}{|c|}{ Administration route } & $\begin{array}{l}\text { Product } \\
\text { Actiq }\end{array}$ & $\begin{array}{l}\text { Dose tested } \\
(\mu g) \\
800 \\
800\end{array}$ & $\begin{array}{l}\text { Cmax } \\
(\mu g / l, \text { mean }) \\
1.0 \\
1.3\end{array}$ & $\begin{array}{l}\mathrm{T}_{\max } \\
\text { (min, median) } \\
120 \\
91\end{array}$ & $\begin{array}{l}\text { Bioavailability (\%) } \\
\text { Total } \\
47\end{array}$ & $\begin{array}{l}\text { Gastrointestinal } \\
25\end{array}$ & $\begin{array}{l}\mathrm{T}_{1 / 2} \\
\text { (h, terminal elimination) } \\
15.3 \\
18.3\end{array}$ & $\begin{array}{l}\text { Reference } \\
\text { Vasisht }^{5} \\
\text { Darwish }^{7}\end{array}$ \\
\hline & Buccal & Breakyl & 800 & 1.3 & 90 & 71 & 20 & 19.0 & Vasisht $^{6}$ \\
\hline & Buccal & Effentora & 400 & 1.0 & 47 & 65 & 17 & 14.4 & Darwish $^{7}$ \\
\hline & & & 800 & 1.7 & 60 & & & 14.4 & Vasisht $^{5}$ \\
\hline \multirow[t]{3}{*}{ Nasal } & With pectin & PecFent & 100 & 0.4 & 20 & NA & NA & 21.9 & Davis $^{3}$ \\
\hline & No pectin & Instanyl & 100 & 1.0 & 11 & $>90$ & NA & 3.0 & Christrup $^{4}$ \\
\hline & & & & 0.6 & 12 & & & & Kaasa ${ }^{10}$ \\
\hline
\end{tabular}

products, median $T_{\text {max }}$ varied between the sublingually delivered tablet, the buccal tablet and the transmucosal administration routes, where the first one was the fastest and the last one the slowest. In all studies, interindividual ranges in $\mathrm{T}_{\max }$ were quite large (range 30-240 min). ${ }^{5-9}$ Also, the terminal half life of fentanyl seems to vary between the various preparations, although this value depends on the moment of pharmacokinetic measurement. Unfortunately, most studies were done in small groups of healthy people $(n=12-48)$. One study was performed in a group of just eight patients with metastatic cancer. ${ }^{9}$

Nasally delivered fentanyl has different characteristics compared with oromucosally delivered products. Median $\mathrm{T}_{\max }$ is much shorter, varying between 11 and 20 min. ${ }^{4-10}$ There is also a much smaller interindividual range compared with the oromucosally delivered products.

Elimination of fentanyl by hepatic metabolism is slow compared with absorption and distribution of fentanyl. Following the absorption phase, distribution to tissue may contribute to the relatively short half life of $3 \mathrm{~h}$, as reported for the Instanyl nasal spray. Thereafter, slow metabolism and redistribution may result in longer terminal half lives of $5-22 \mathrm{~h}$, as reported for the other products (table 1).

\section{Clinical aspects}

The four administration routes of immediate release products of fentanyl have been compared with placebo in clinical efficacy studies. All studied drugs were superior to placebo in terms of faster pain relief. Between 15 and 30 min after administration, a difference in pain intensity between the fentanyl products and placebo was found, as shown in figure $1 .^{11}$ Two studies compared oromucosally delivered fentanyl with other immediate release opioids using a randomised, double blind study design. ${ }^{1213}$ Both studies showed more pain relief for the

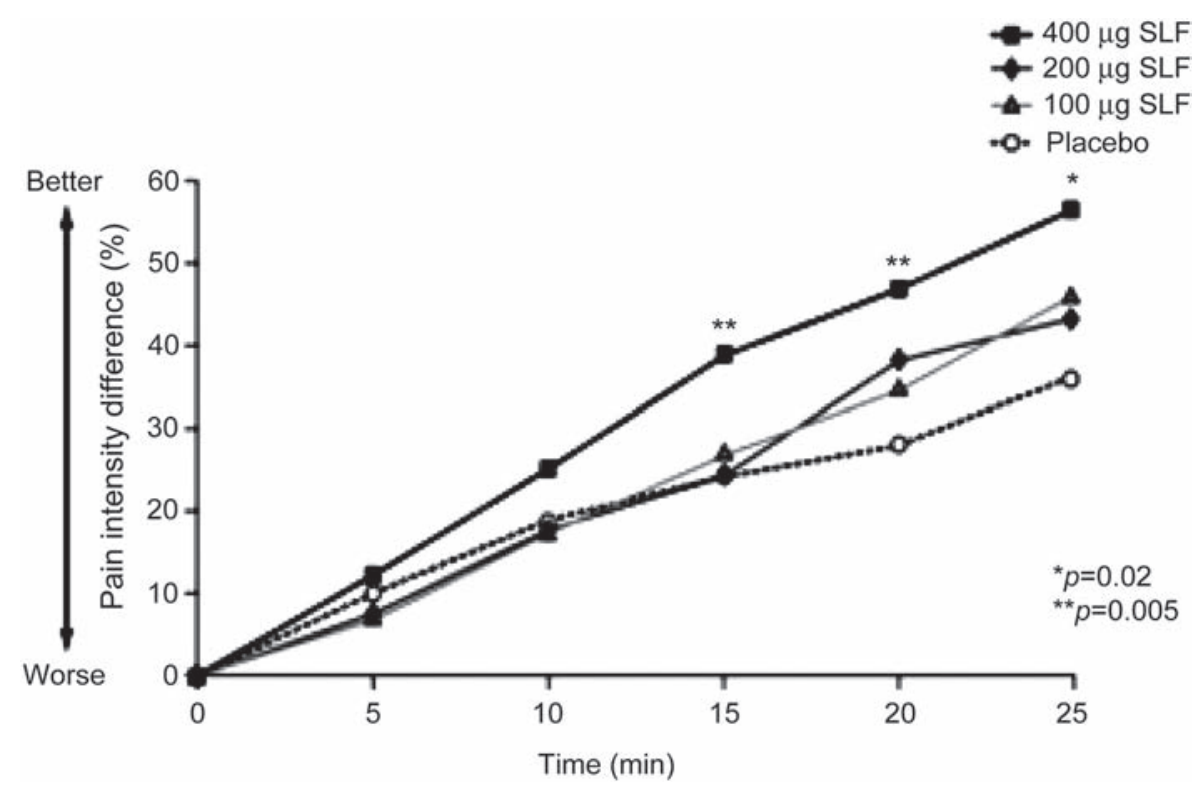

Figure 1 Mean pain intensity difference versus time following administration of sublingual fentanyl (SLF) 100, 200 or $400 \mu \mathrm{g}$ compared with placebo. This figure was obtained with permission from Lennernäs and colleagues. ${ }^{11}$

groups using oromucosal immediate release fentanyl formulations than for patients using morphine or oxycodon. Another study compared nasally delivered fentanyl with immediate release morphine and also found a significantly larger decrease in pain from 15 min after delivery for fentanyl. ${ }^{14}$ Unfortunately, until now, there have been no double blind, randomised studies that have compared immediate release fentanyl products with each other.

Because of the long elimination time compared with the short absorption time of the fast acting fentanyl products, there is a risk of accumulation of fentanyl. This could lead to signs of overdosing such as respiratory depression, bradycardia, hypotension and nausea. However, this has not been reported to be relevant in the reported clinical trials, possibly due to limited and controlled fentanyl use in these studies. $^{8-15}$ In clinical practice, the risk of accumulation needs attention, although it is advised that doses be titrated carefully.

\section{Conclusions and perspectives}

Nasally delivered fentanyl products are favourable to other immediate release products in terms of pharmacokinetic properties. Because of its short $T_{\max }$, fentanyl spray resembles the efficacy of intravenous fentanyl (the golden standard) most closely of all available products.

Oromucosally delivered preparations result in slower peak concentrations, and therefore time to effect may not be soon enough in all patients with breakthrough pain. Nevertheless, a significant part of fentanyl is absorbed transmucosally and will provide adequate serum concentrations within the first hour after administration. In line with these data, clinical trials have demonstrated a modest benefit in breakthrough pain relief over placebo and oral opioids. As interpatient variability is relatively large, with a wide range in $T_{\max }$, it may be useful to try different oromucosal preparations in clinical practice. In addition, patient preferences may play an important 
role in the selection of the optimal product for the individual patient. For patients with relatively 'predictable' breakthrough pain, such as the pain which occurs when starting daily activities, oromucosally delivered fentanyl preparations can be useful additions to the range of oral opioid products. It is likely that clear instructions to patients and nurses, and titration to the optimal dose for the individual patient, will improve clinical effectiveness.

Considering the available publications, it is important to stress that the vast majority of pharmacokinetic data were obtained from healthy adults or patients with minimal morbidity, and of normal weight, with normal renal and liver function, and without interacting co-medication. Pharmacokinetics may differ in patients with malignant disease. Moreover, these patients are often cachectic, use multiple drugs and can have organ failure. Apart from this, specific characteristics-for example, the amount of saliva-may affect the speed of the dissolving oromucosally delivered preparations and may therefore be a relevant factor in absorption and bioavailability. A dry mouth is a common side effect of opioids and is rather prevalent in the target population. Although optimal schedules for the use of immediate release opioids in these specific patients remain to be established, the immediate release fentanyl preparations have (substantially) increased our possibilities of treating breakthrough pain in cancer.

Competing interests None.

Contributors All authors contributed scientifically to the manuscript and approved the final version.

\section{Key messages}

Various immediate release fentanyl products have been developed recently for the treatment of breakthrough cancer pain: products for oromucosal and nasal delivery. These products differ with respect to bioavailability and time to reach maximal plasma levels. Interindividual variability is large, even in healthy controls, especially for the oromucosal products. All drugs have proven to provide fast relief of breakthrough cancer pain in placebo controlled studies but insights into factors predicting a good response for the different products in individual patients have to be awaited.

Provenance and peer review Commissioned; externally peer reviewed.

\section{References}

1. Greco MT, Corli $\bigcirc$, Montanari M, et al.; Writing Protocol Committee; Cancer Pain Outcome Research Study Group (CPOR SG) Investigators. Epidemiology and pattern of care of breakthrough cancer pain in a longitudinal sample of cancer patients: results from the Cancer Pain Outcome Research Study Group. Clin J Pain 2011;27:9-18.

2. Portenoy RK, Payne D, Jacobsen P. Breakthrough pain: characteristics and impact in patients with cancer pain. Pain 1999:81:129-34.

3. Davis MP. Fentanyl for breakthrough pain: a systematic review. Expert Rev Neurother 2011;11:1197-216.

4. Christrup LL, Foster D, Popper LD, et al. Pharmacokinetics, efficacy, and tolerability of fentanyl following intranasal versus intravenous administration in adults undergoing third-molar extraction: a randomized, double-blind, doubledummy, two-way, crossover study. Clin Ther 2008;30:469-81.

5. Vasisht N, Gever LN, Tagarro I, et al. Formulation selection and pharmacokinetic comparison of fentanyl buccal soluble film with oral transmucosal fentanyl citrate: a randomized, open-label, single-dose, crossover study. Clin Drug Investig 2009;29:647-54.
6. Vasisht N, Gever LN, Tagarro I, et al. Single-dose pharmacokinetics of fentanyl buccal soluble film. Pain Med 2010;11:1017-23.

7. Darwish M, Kirby M, Robertson P Jr, et al. Absolute and relative bioavailability of fentanyl buccal tablet and oral transmucosal fentanyl citrate. J Clin Pharmacol 2007;47:343-50.

8. Lister N, Warrington S, Boyce M, et al. Pharmacokinetics, safety, and tolerability of ascending doses of sublingual fentanyl, with and without naltrexone, in Japanese subjects. J Clin Pharmacol 2011:51:1195-204.

9. Lennernäs B, Hedner T, Holmberg M, et al Pharmacokinetics and tolerability of different doses of fentanyl following sublingual administration of a rapidly dissolving tablet to cancer patients: a new approach to treatment of incident pain. Br J Clin Pharmacol 2005;59:249-53.

10. Kaasa S, Moksnes K, Nolte T, et al. Pharmacokinetics of intranasal fentanyl spray in patients with cancer and breakthrough pain. J Opioid Manag 2010;6:17-26.

11. Lennernäs $\mathbf{B}$, Frank-Lissbrant I, Lennernäs $\mathrm{H}$, et al. Sublingual administration of fentanyl to cancer patients is an effective treatment for breakthrough pain: results from a randomized phase II study. Palliat Med 2010;24:286-93.

12. Portenoy RK, Burton AW, Gabrail N, et al.; Fentanyl Pectin Nasal Spray 043 Study Group. A multicenter, placebo-controlled, double-blind, multiple-crossover study of fentanyl pectin nasa spray (FPNS) in the treatment of breakthrough cancer pain. Pain 2010;151:617-24

13. Coluzzi PH, Schwartzberg L, Conroy JD, et al. Breakthrough cancer pain: a randomized trial comparing oral transmucosal fentanyl citrate (OTFC) and morphine sulfate immediate release (MSIR). Pain 2001;91:123-30.

14. Ashburn MA, Slevin KA, Messina J, et al. The efficacy and safety of fentanyl buccal tablet compared with immediate-release oxycodone for the management of breakthrough pain in opioid tolerant patients with chronic pain. Anesth Analg 2011;112:693-702.

15. Fallon M, Reale C, Davies A, et al.; Fentanyl Nasa Spray Study 044 Investigators Group. Efficacy and safety of fentanyl pectin nasal spray compared with immediate-release morphine sulfate tablets in the treatment of breakthrough cancer pain: a multicenter, randomized, controlled, double-blind, double-dummy multiple-crossover study. J Support Oncol 2011;9:224-31. 